\title{
The Weird Detector: Flagging periodic, coherent signals of arbitrary shape in time series photometry
}

\author{
Adam Wheeler $^{1 \star}$ and David Kipping ${ }^{1}$ \\ ${ }^{1}$ Dept. of Astronomy, Columbia University, $550 \mathrm{~W}$ 120th Street, New York NY 10027
}

Accepted . Received ; in original form

\begin{abstract}
By design, model-based approaches for flagging transiting exoplanets in light curves, such as boxed least squares, excel at detecting planets with low $\mathrm{S} / \mathrm{N}$ at the expense of finding signals that are not well described by the assumed model, such as self-lensing binaries, disintegrating or evaporating planets, or planets with large rings. So far, such signals have typically been found through visual searches by professional or citizen scientists, or by inspection of the photometric power-spectra. We present a nonparametric detection algorithm, for short duty-cycle periodic signals in photometric time series based on phase dispersion minimization. We apply our code to 161,786 Kepler sources and detect 18 new periodic signals consistent with heartbeat binaries/planets, 4 new singly-transiting systems, and 2 new doubly-transiting systems. We show that our code is able to recover the majority of known Kepler objects of interest (KOIs) to high confidence, as well as more unusual events such as Boyajian's star and a comet passing through the Kepler field. Nonparametric signal-flagging techniques, such as the one presented here, will become increasingly valuable with the coming data from TESS and future transit surveys as the volume of data available to us exceeds that which can be feasibly examined manually.
\end{abstract}

Key words: methods: data analysis; stars: planetary systems

\section{INTRODUCTION}

The photometric transit method has emerged as the most successful technique for detecting new exoplanets. At the time of writing, the Kepler Mission alone has brought in a haul of over 4500 transiting planet candidates, of which the majority have now been confirmed (see the NASA Exoplanet Archive; Akeson et al. 2013). With TESS expected to detect some $10^{4}$ new examples (Sullivan et al. 2015; Bouma et al. 2017; Barclay et al. 2018; Ballard 2018; Huang et al. 2018) and future surveys looking set to pull in even larger yields (e.g. see Rauer et al. 2014; Jacklin et al. 2015; Cortes \& Kipping 2018), the transit method looks to remain a critical tool to exoplanetary science for many years to come.

The algorithm used to detect new transiting planet candidates varies between missions and teams. The most frequently cited method is the Boxed Least Squares (BLS) algorithm (Kovács et al. 2002), which behaves as an optimal detector when the signal is i) strictly-periodic ii) boxshaped iii) in the presence of Gaussian noise. Real transits show limb darkening curvature (Knutson et al. 2007) and time-integration smoothing (Kipping 2010), but BLS remains nearly optimal even when the light curve becomes

^ E-mail: a.wheeler@columbia.edu somewhat U-shaped. Many alternative algorithms have been proposed to BLS, which are usually designed to tackle cases where either assumption i) or iii) are relaxed. As far as we can tell, no transit detection algorithms have been explicitly designed with the objective of relaxing assumption ii), however.

As an example, the Quasi-Periodic Automated Transit Search Algorithm (QATS) assumes that transits are trapezoidal-like and in the presence of Gaussian noise, but can be non-periodic (Carter \& Agol 2013). As another example, time-correlated noise structure has been known to affect transit detection since Pont et al. (2006), and is most commonly tackled by pre-whitening and detrending of the time series prior to searching (e.g. Tamuz et al. 2005; Kovács et al. 2005; Jenkins et al. 2010; Guterman et al. 2015), although recently deep learning approaches have attacked the problem without this filtering step (e.g. see Pearson et al. 2018; Shallue \& Vanderburg 2018; Zucker \& Giryes 2018). In all of the above, an underlying assumption is that transits are expected to have a shape consistent with a trapezoid.

Irregularly shaped transits, including asymmetric and multi-dip events, can emerge in a variety of plausible astrophysical scenarios. Planetary rings (Barnes \& Fortney 2004; Zuluaga et al. 2015) and moons (Kipping 2011) are examples we'd expect based on the Solar System planets, causing tran- 
sits with an irregular morphology due to the overlap with another occulter. Disintegrating planets (Rappaport et al. 2012), photoevaporation (Vidal-Madjar et al. 2003), circumstellar material (Vanderburg et al. 2015), proto-satellite disks (Mamajek et al. 2012), bow shocks (Llama et al. 2013), gravity darkening (Barnes 2009), planetary oblateness (Seager \& Hui 2002), atmospheric refraction (Hui \& Seager 2002), and even extreme orbital eccentricities (Kipping 2008) have all been argued to be other plausible astrophysical effects which could distort the transit. Distorted transits have even been argued to be a possible means of detecting advanced civilizations (Arnold 2005; Korpela et al. 2015; Kipping \& Teachey 2016). While small perturbations to the transit should not greatly impact BLS's sensitivity, highly irregular transits require another approach. Although there are presently only a handful of known examples of these "weird" transits, with perhaps the most dramatic being that of Boyajian's star (Boyajian et al. 2016), they represent some of the most scientifically rich objects to date.

Arguably the most successful approach to date for searching for weird transits has been through the citizen science Planet Hunters program (Fischer et al. 2012). This program demonstrates that human beings certainly have the ability to successfully identify unusual shaped signals, although any approach using humans will face challenges with scalability and statistical testing. An software-based solution could overcome such hurdles although to date there has been little attention devoted to this issue. In this work, we therefore aim to address this problem by presenting a so-called weird detector for photometric time series.

Our method is related to phase dispersion minimization (PDM), variants of which have been employed for decades in other astronomical contexts. To the best of out knowledge, a variant of PDM appeared first in Whittiker \& Robinson (1926), a general work on data analysis. A similar idea was employed in Lafler \& Kinman (1965), which introduced the used of "string-length" methods, in their case to compute the periods of RR Lyrae stars. Similar methods were developed again in Jurkevich (1971), Warner \& Robinson (1972), and Stellingwerf (1978), from which the term "phase dispersion minimization" arises. Such methods used to supersede the discrete Fourier transform and the Lomb-Scargle periodogram (Lomb 1976; Scargle 1982) for determining the period of variable stars and stellar rotation because they do not require the evaluation of trigonometric functions and were thus less expensive to run given the limited computational resources of the time (Kovács et al. 2002). More recently, Plavchan et al. (2008) applied a similar algorithm to find both variable stars and transiting exoplanets in data from the Two Micron All Sky Survey (2MASS) (Skrutskie et al. 2006). Parks et al. (2014) also applies a "binless" PDM variant to search for variable stars in 2MASS data.

In Section 2, we describe our algorithmic solution (dubbed weirddetector; Julia implementation available at this URL) and the details behind the code's operation and assumptions. In Section 2.4, we explore the performance of our code in application to the Kepler data, including numerous new detections. Finally, we discuss the scalability, future improvements and applications of the weird detector in Section 5.

\section{METHODOLOGY}

\subsection{Principles and Assumptions}

In designing an algorithm to detect irregular signals, one is faced with the fundamental question of what one wishes to optimize for. In a least squares regression problem, for example, the cost function is the sum of the residuals (optionally weighted by their uncertainty) squared, where the residuals are computed against some parametric model. In the case of BLS, which is an example of a least squares problem, the model employed is a periodic boxcar function defined by a period, phase, depth and duration (Kovács et al. 2002). For ordinary transits, this is a well-motivated simplification, but for signals with morphology deviating strongly from an inverted boxcar transit, the mismatch between model and data is problematic.

One could imagine using a variant of the BLS code based on nested transits for detecting rings and moons, or a version incorporating a skewed transit model in order to cope with disintegrating planets. But each code would struggle with other effects and both would be bespoke algorithms rather than generalized weird detectors. We quickly decided that proposing any kind of parametric function to describe the transit morphology would be limiting, in that our code could then only detect signals which could be described by said function. Ultimately, we wanted an algorithm which could handle the unexpected and even have the ability to detect signals previously unimagined. At the same time, the problem of detecting any and all effects optimally is both illdefined and formally intractable. Some underlying assumptions are always necessary.

In this work, our formal assumptions are that the weird signals we seek are strictly periodic and in the presence of Gaussian noise - i.e. assumptions i) and iii) used by BLS. We make only minimal assumptions about the signal's shape. By virtue of the strict periodicity assumption, the signal is implicitly not time varying and thus repeats each epoch. We highlight that ordinary planetary transits also satisfy this condition and produce coherent signals in folded photometry, but more generally any repeating signal will do so, be it a lensed black hole or an orbiting alien megastructure. Boyajian's star satisfies these assumptions only approximately, which means that we are limited to detecting its strictly periodic analogs.

We therefore loosely state that our objective function should identify signals that are both coherent and have a transit-like morphology, in the sense of being narrow, rather than extended across an entire phase. This is primarily to avoid flagging brightness modulation stellar from rotation in the presence of starspots. Such signals should be associated with high power, whereas pure Gaussian noise would produce low or ideally negligible power. It should be noted that already at this stage, it can be stated that weirddetector must have lower sensitivity to planetary transits than BLS. This is because BLS includes additional information about the signal that our algorithm does not - the shape of the expected events. Because of weirddetector's much weaker assumptions about signal shape, it has inferior sensitivity for box-like signals. This is a necessary trade-off for any weird detector though, imposed by the differing cost functions. 


\subsection{Quantifying Coherence and Morphology}

Having established that we wish to detect coherent signals, the next step is to identify an approach for achieving this goal. Let us assume that the number of epochs constituting a folded signal is large and that any nuisance signals (e.g. stellar variability) have been removed. We would expect that each epoch's phase curve is self-similar to the others. In other words, the dispersion at any phase location is small. We might imagine, then, that if we folded our time series on many trial periods we could identify the correct period by seeing which one exhibits the smallest phase dispersion.

For each period in our search grid, weirddetector first folds the data to construct a phase curve. We then use a rolling mean over the fluxes (weighted by their photometric errors) with a constant phase window of width $\Delta \phi$ to construct a smoothed phase curve (hereafter a candidate signal; e.g. Figure 1a). Specifically, for each point $i$ in a phase curve with $N$ points, it computes the value of the candidate signal

$F_{i}^{\prime}=\frac{\sum_{j=1}^{N} F_{j} w_{i j}}{\sum_{j=1}^{N} w_{i j}}$

where

$w_{i j}=\sigma_{i}^{-2} \Theta\left(\Delta \phi-\left|\phi_{i}-\phi_{j}\right|\right)$.

Here $\phi_{i}, F_{i}$, and $\sigma_{i}$ are the phase, flux, and photometric error, respectively, of the $i$ th point and $\Theta$ is the Heaviside step function. For convienience, we define the relative flux as $\delta F=F / \bar{F}-1$.

For each candidate signal, weirddetector computes two statistics: $\chi^{2}$, which reflects how coherent the phase curve is, and $\kappa$, which quantifies the morphology of the candidate signal. Specifically,

$\chi^{2}=\sum_{i=1}^{N}\left(\frac{F_{i}-F_{i}^{\prime}}{\sigma_{i}}\right)^{2}$.

Since the values of $F_{i}^{\prime}$ are calculated directly from the values of $F_{i}$ themselves, this describes the scatter (dispersion) of flux values at a constant phase. This is what our method has in common in with the family of PDM techniques. The other statistic, $\kappa$, is the the fourth standardized moment, or kurtosis, of the distribution of flux values in the candidate signal (see Figure 1b). Specifically,

$\kappa=\frac{1}{N} \sum_{i=1}^{N}\left(\frac{F_{i}^{\prime}-\mu}{\sigma}\right)^{4}$

where $\mu$ and $\sigma$ are the mean and standard deviation, respectively, of the distribution of fluxes in the candidate signal. Kurtosis quantifies roughly the "tailedness" of a distribution; flux distributions of candidate signals with low duty cycles will have a dominant mode around $\delta F=0$, with outliers on one or both sides caused by the signal. Fig $1 d$ demonstrates that while both $\kappa$ and $\chi^{2}$ peak at the correct period, a LombScargle periodogram does not pick out the signal, since it is not well-described by a sinusoid.

The fourth standardized moment is the lowest that will work for our purposes, since the third (skewness) is insensitive to $F_{i}^{\prime}$ drawn from symmetrical distributions, and the first and second standardized moments are identically zero and one, respectively. The standard deviation of the fluxes in
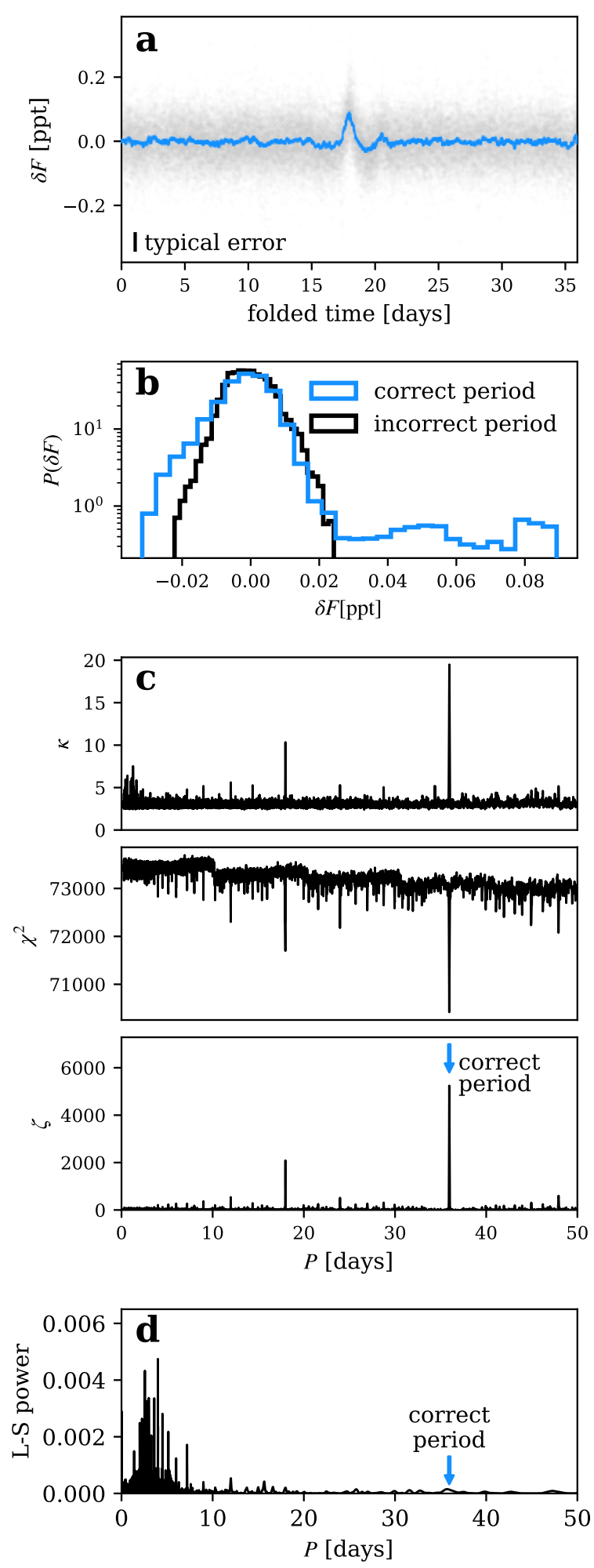

Figure 1. a: The phase curve of KIC 8694536 folded on $P=35.95$ days with the candidate signal in blue. b: Histograms of the distribution of $f_{i}^{\prime}$ when the light curve is folded on the correct $(P=35.95$ days $)$ and incorrect $(P=30$ days $)$ period. Note the presence of heavy tails on the distribution from folding on the correct period. c: $\kappa, \chi^{2}$, and $\zeta$, which combines the two, as a function of period. d: The Lomb-Scargle periodogram for the same signal, which does not have significant power at the correct period. 
the candidate signal (i.e. the unstandardized centered second moment) will pick out signals with large phase-integrated amplitude, but will not strongly prefer short duty-cycle signals over those more extended in phase, such as the flux modulations from rotating stars (see Figure 2a). Conversely, to the extent that the phases assigned to each point are random (i.e. the period is wrong) the distribution of fluxes in the candidate signal will be roughly Gaussian, since they are defined as the average of many randomly chosen flux measurements.

From these quantities, we calculate $\kappa^{\prime} \Delta \chi^{2}$ at every period, where $\kappa^{\prime}=\kappa-3$ is excess kurtosis, kurtosis less that of a Gaussian, and $\Delta \chi^{2}$ is the local decrease in $\chi^{2}$ (see below for details). We chose to use the product, rather than a weighted sum of these quantities for two reasons. First, we wish to flag periods at which both statistics deviate from their baseline value. This is the same reason we use $\kappa^{\prime}$ rather than $\kappa$ and $\Delta \chi^{2}$ rather than $\chi^{2}$. A null value of one statistic will "cancel out" a non-null value of the other (see, for example, Figure 2c). Second, a sum of $\Delta \chi^{2}$ and $\kappa^{\prime}$ would require choosing relative weights, a tuning parameter that we prefer to avoid. Calculating this requires some model of the "signal-free" or "baseline" relationship between $\chi^{2}$ and period. For uniformly spaced data (such as Kepler light curves), we found that $\chi^{2}(P)$ takes discontinuous steps whenever the duration of the smoothing kernel is a multiple of $\Delta t$, the light curve cadence. This occurs whenever $P$ is a multiple of $\Delta t / \Delta \phi$. For our application to Kepler, this is

$$
\frac{\Delta t}{\Delta \phi}=\frac{0.02042 \mathrm{~d}}{0.002}=10.208335 \mathrm{~d} \text {. }
$$

We fit a piecewise-linear function with discontinuities at multiples of $\Delta t / \Delta \phi$ to obtain $\widehat{\chi^{2}}(P)$, then calculated $\Delta \chi^{2}(P)=$ $\widehat{\chi^{2}}(P)-\chi^{2}(P)$. As mentioned later in section 2.4 , this procedure does not correct perfectly for the discontinuous behaviour in $\chi^{2}$. A better understanding of the mechanism causing these discontinuities would presumably suggest a better way to correct for or prevent them, but we were unable to determine their source and will leave its determination to future work.

For each light curve, we construct a scrambled time series by randomly re-assigning times to flux-uncertainty pairs. This preserves the distribution of fluxes in the time series while erasing all patterns in the time domain. We use weirddetector to calculate a periodogram from the scrambled data, from which we calculating the moving standard deviation, which we use to get the merit function (Figure 1c)

$$
\zeta(P)=\frac{\kappa^{\prime}(P) \Delta \chi^{2}(P)}{\sigma(P)}
$$

where $\sigma(P)$ is the standard deviation of $\kappa^{\prime} \Delta \chi^{2}$ values in a small window surrounding $\mathrm{P}$ in the periodogram constructed from scrambled data (we used a window of width 401 points centered around $\mathrm{P}$, which spans roughly $0.96 P$ to $1.04 P$ ). This quantity tracks, but is not equal to, the signal-to-noise ratio $(\mathrm{S} / \mathrm{N}$ ) at each period (strictly $\mathrm{S} / \mathrm{N}$ would be given by the $\Delta \chi^{2}$ term alone). While the $\zeta$ might be interpreted as a false-alarm probability (FAP) or p-value under certain assumptions, we caution the reader against this. Real light curves have an abundance of periodic structure from stellar rotation and variability, and the null hypothesis and mani-
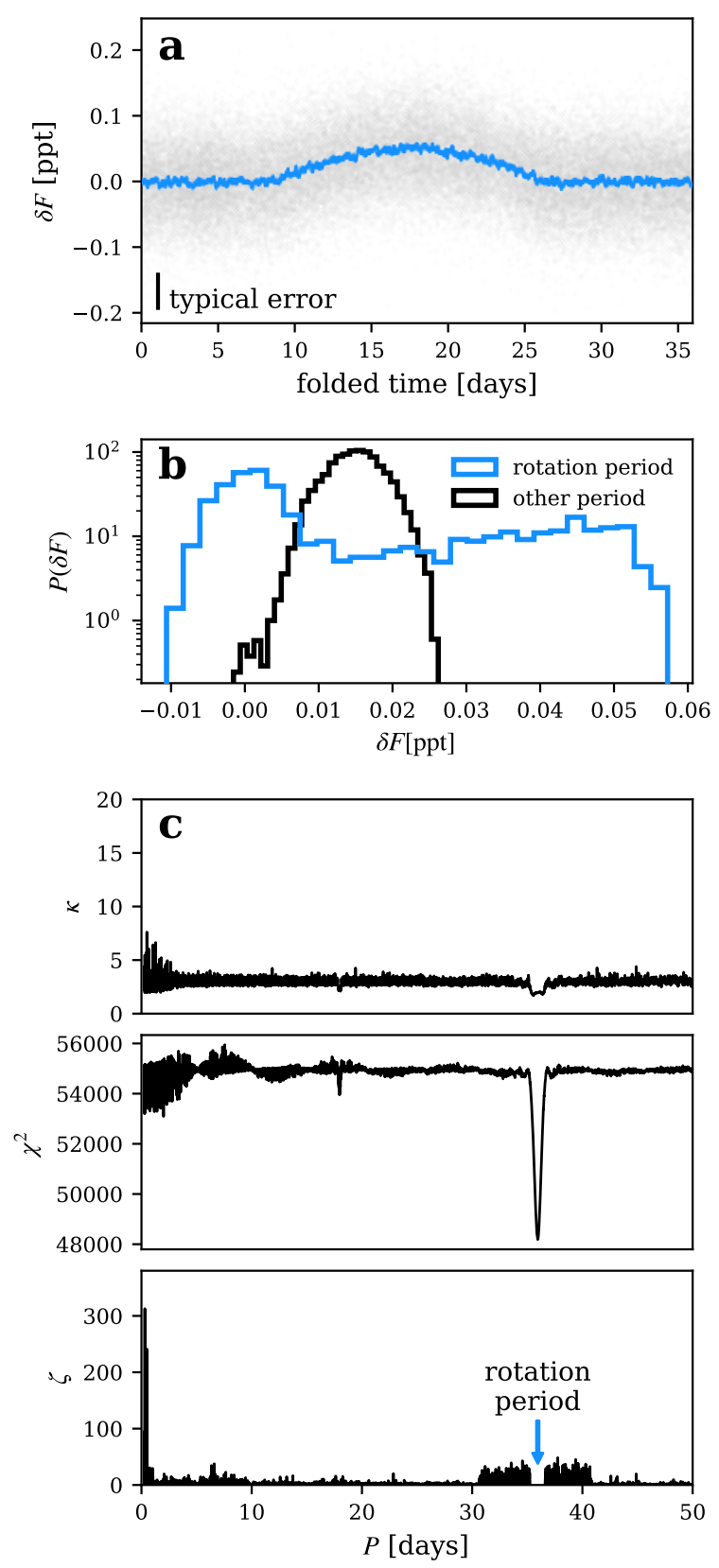

Figure 2. Same as Figure 1, but for a simulated ideal rotator (i.e. a pure sine wave with all negative values set to 0 ). Note that the weirddetector periodogram does not have a peak corresponding to the injected rotation signal. a: The phase curve of the simulated data folded on $P=35.95$ days with the candidate signal in blue. b: Histograms of the distribution of $f_{i}^{\prime}$ when the light curve is folded on the correct $(P=35.95$ days $)$ and incorrect $(P=30$ days $)$ period. c: $\kappa, \chi^{2}$, and $\zeta$, which combines the two, as a function of period.

festly false in almost all cases. We calculate $\zeta$ not to obtain a FAP for each detection, but to provide a way of ranking peaks from different periodograms. An empirical investigation of the relationship between false-positive rate and truepositive rate is presented in Section 3. Note that $\zeta$ (as well as 
$\Delta \chi^{2}$ and $\kappa$ individually) picks out the correct period, while a Lomb-Scargle periodogram (Figure 1) does not.

\subsection{Aliasing}

An inevitable outcome of using a folding technique with such a flexible model is spurious peaks in the periodogram at rational fractions and multiples of the "true" period of a signal (Figure 3). These peaks are present in both $\chi^{2}$ and $\kappa$, since they come from the construction of the candidate signal itself, rather than the operations performed upon it. We see these peaks in both synthetic and real data.

It's worth noting that this is a different phenomenon from aliasing in the context of the discrete Fourier transform, which is caused by finite sampling of a continuous signal. It's also distinct from the phenomenon of harmonics seen in the power spectra of non-sinusoidal signals.

The height of each aliased peak decreases roughly with the denominator in the reduced rational form of it's period in units of $P_{\text {true }}$, in other words it decreases with $n$, where

$\frac{m}{n}=\frac{P_{\text {flagged }}}{P_{\text {true }}}$

is the reduced rational form of the ratio between flagged and true periods. Examination of phase-folded light curves, shows that higher values of $n$ correspond to a loweramplitude aliased signal and thus a smaller peak in the periodogram. This can be seen in Figure 3, which shows the periodogram for Kepler-1b with aliases labelled. Because of the difficulty of automatically disambiguating between aliases and true periods, we don't attempt to flag multiple signals per light curve.

\subsection{Application to Kepler data}

We ran weirddetector on the instrumentals-removed PDCSAP fluxes on all long-cadence light curves in Kepler DR25 with at least twelve of 16 quarters present, not counting Q0 and Q17, which we don't use (161,786 stars). We performed automatic outlier rejection by removing all points more than $5 \sigma_{\mathrm{MAD}}$ away from the median within an 11 point window, where $\sigma_{\text {MAD }}$ is the standard deviation estimated by the mean absolute deviation:

$\sigma_{\mathrm{MAD}}=1.4826 \times \operatorname{median}\left(\left|F_{i}-\operatorname{median}(\mathbf{F})\right|\right)$.

We also removed all points with non-zero quality flags and split each time series into segments at quarter boundaries and anywhere with a missing-data gap of 0.3 days or greater. Within each segment, we used linear interpolation to fill in any missing data, then detrended with a 2-day (roughly 99 cadences) temporally-windowed rolling median to remove long duty-cycle signals and trends. After detrending, we removed the interpolated points.

To compute periodograms for each star in our input catalog, we folded on a grid of 26,492 period values from 0.25 days up to 50 days, with sampling uniform in $\log P$ (motivated by experiments with synthetic data showing that periodogram peak width increases thusly). Although we are primarily looking for signals with periods in this range, weirddetector is still sensitive to signals with shorter or longer periods if they have a strong alias in our period grid. We constructed our candidate signals with $\Delta \phi=0.002$ (see Equation 2), which corresponds to roughly 120 points in a Kepler light curve with no missing data. The number of points in a constant-width phase bin of a phase curve is not dependant on the folding period $P$, since folding amounts roughly to a reordering of points.

We consider only the most significant peak from each light curve in order to avoid the problem of disentangling aliases from independent signals. We make a few cuts in order to remove as many spurious signals as possible (see Figure 4). First, the 200 period values most frequently flagged are eliminated on the grounds that they are likely an artifact of the data analysis. These period values represent only $0.75 \%$ of the period grid over which we search. The number of removed periods was somewhat ad-hoc, but we manually examined random signals from this population to ensure that they were contaminated by spurious artifacts of the Kepler cadence. Second we remove any signals with $P<2$ days or with $P$ too close to a discontinuity in $\widehat{\chi^{2}}$ (specifically, $P /(1 \mathrm{~d})$ in any of $(10,10.3),(20.2,20.5),(30.4,30.7)$, or $(40.6,40.9))$.

We cross-matched the resulting catalog by Kepler input catalog number (KIC) with the Villanova eclipsing binary catalog (Kirk et al. 2016; Abdul-Masih et al. 2016), Kepler DR25 KOIs and TCEs ${ }^{1}$ (Thompson et al. 2018), the candidates from (Huang et al. 2013), the long-period candidates from the wavelet-based search of Foreman-Mackey et al. (2016) and planet hunters (Wang et al. 2015), and the ultra-short period planets in Sanchis Ojeda et al. (2015). We cross-match with both candidates and false positives from all catalogs. Finally, we throw out any signal with $\kappa<5$ on the grounds that they are very likely to be long duty-cycle signals like stellar rotation. After removing these KICs, we were left with with 4,426 flagged signals.

\section{RECOVERY OF OTHER CATALOGS}

As a way of quantifying the weirddetector's performance, we analyzed its performance as a binary classifier of Kepler light curves. While injection-recovery testing is commonly used to test new detection algorithms of various types, we avoided this approach since our goal was not to recover a specific signal, and there is no obvious signal type to inject. Framing our algorithm as a binary classifier relies on the fact that we attempt to flag at most one signal per light curve. We consider a signal flagged if it's periodogram contains a peak with $\zeta>\zeta_{\text {threshold }}$, and we evaluate our recovery of signals in other catalogs as we vary $\zeta_{\text {threshold }}$ A signal is considered an base-truth (expected) positive if it is in any of the catalogs with which we cross-matched (Kirk et al. 2016; Abdul-Masih et al. 2016; Thompson et al. 2018; Huang et al. 2013; Sanchis Ojeda et al. 2015), save Wang et al. (2015) and Foreman-Mackey et al. (2016), which include nonperiodic signals. In this context, a false-positive is a distinct concept from a scientific-false positive and has not been manually vetted. Rather, it's a light curve with flagged

\footnotetext{
1 Some of the signals we flagged were TCEs in Kepler DR24, but were removed in DR25. Additionally some are flagged in the Kepler inverted TCE list (Coughlin 2017)
} 


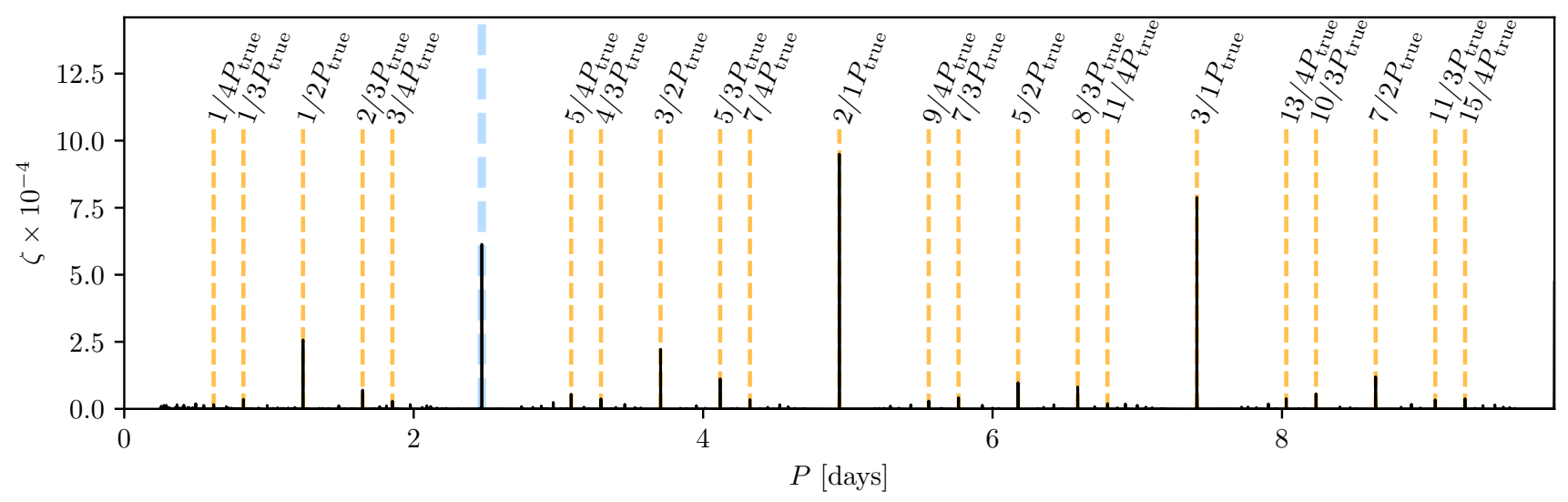

Figure 3. Periodogram generated from light curve of Kepler-1, also known are TrES-2 (O'Donovan et al. 2006), with aliases of Kepler1 b's period down to $n=3$ labelled. The correct orbital period is marked with the thick blue line. The presence of such aliases is why we consider only the highest peak from each periodogram.
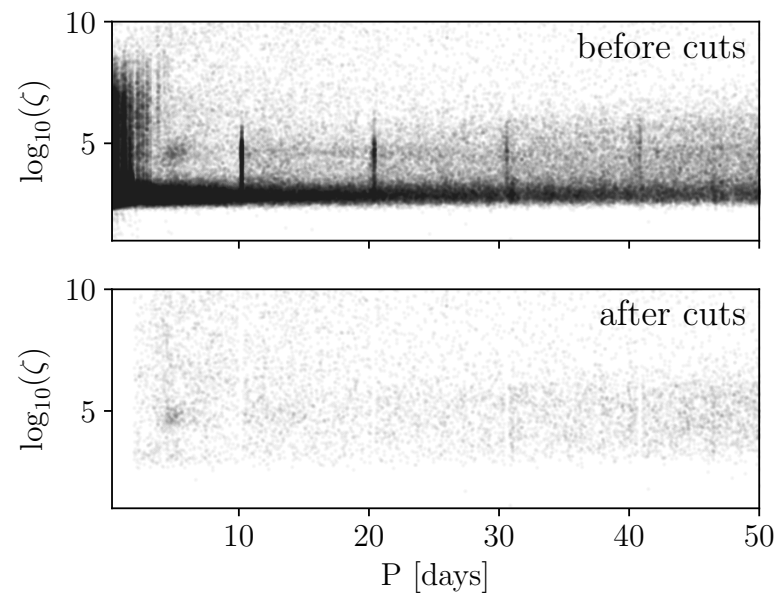

Figure 4. Flagged period values before and after making cuts. The overdensity of signals around $P \approx 5$ days is caused by our approximation of $\widehat{\chi^{2}}$. Manual examination of randomly selected events from this region revealed no unique characteristics.

by weirddetector with no detections in the cross-matched catalogs. Likewise, in this context, a true-positive can be a scientific false-positive if our algorithm flagged a signal that was also flagged but listed as a scientific false-positive in a cross-matched catalog. In other words, we are adopting the approximation that the set of interesting signals (true positives) is exactly the union of the signals in the cross-matched catalogs. Although the majority of these signals are transits or transit candidates and thus better flagged by a modelbased approach, weirddetector would have recovered them equally well if they were inverted or otherwise morphologically perturbed.

Figure 5 shows precision-recall and receiver operating characteristic (ROC) curves for our algorithm as a classifier of Kepler light curves. If we define $Y$ and $\widehat{Y}$ to encode the base-truth and classifier output as

$Y= \begin{cases}1 & \text { signal present } \\ 0 & \text { signal not present }\end{cases}$

and

$\widehat{Y}=\left\{\begin{array}{ll}1 & \text { light curve flagged } \\ 0 & \text { light curve not flagged }\end{array}\right.$,

we can define precision, recall (true positive rate; TPR), and false positive rate (FPR) as

precision $=P(Y=1 \mid \widehat{Y}=1)$,

recall $=\mathrm{TPR}=P(\widehat{Y}=1 \mid Y=1)$,

and

$\mathrm{FPR}=P(\widehat{Y}=1 \mid Y=0)$.

The precision-recall and ROC curves show how these values change and the classification threshold in $\zeta$ is changed. While precision-recall curves are often more informative for needle-in-a-haystack problems where $P(Y=1)$ is very low, their sensitivity to the number of base-truth positives (interesting signals, i.e. $Y=1$ ) in the sample can make them less informative across data sets. The ROC curves quantify the performance of our algorithm in a way that is independent of the fraction of interesting signals. From both curves, it's clear that we are able to recover slightly over half of the signals in the cross-matched catalogs with very minimal false-positive contamination, which we can expect to be true for datasets with a similar fraction of true positives.

\section{NOVEL DETECTIONS}

After filtering these KICs out, most of what remained were artifacts of stellar activity and rotation. We examined all the signals by eye, and identified 52 that didn't appear to be the result of flares, rotation, or otherwise spurious. Additionally, 

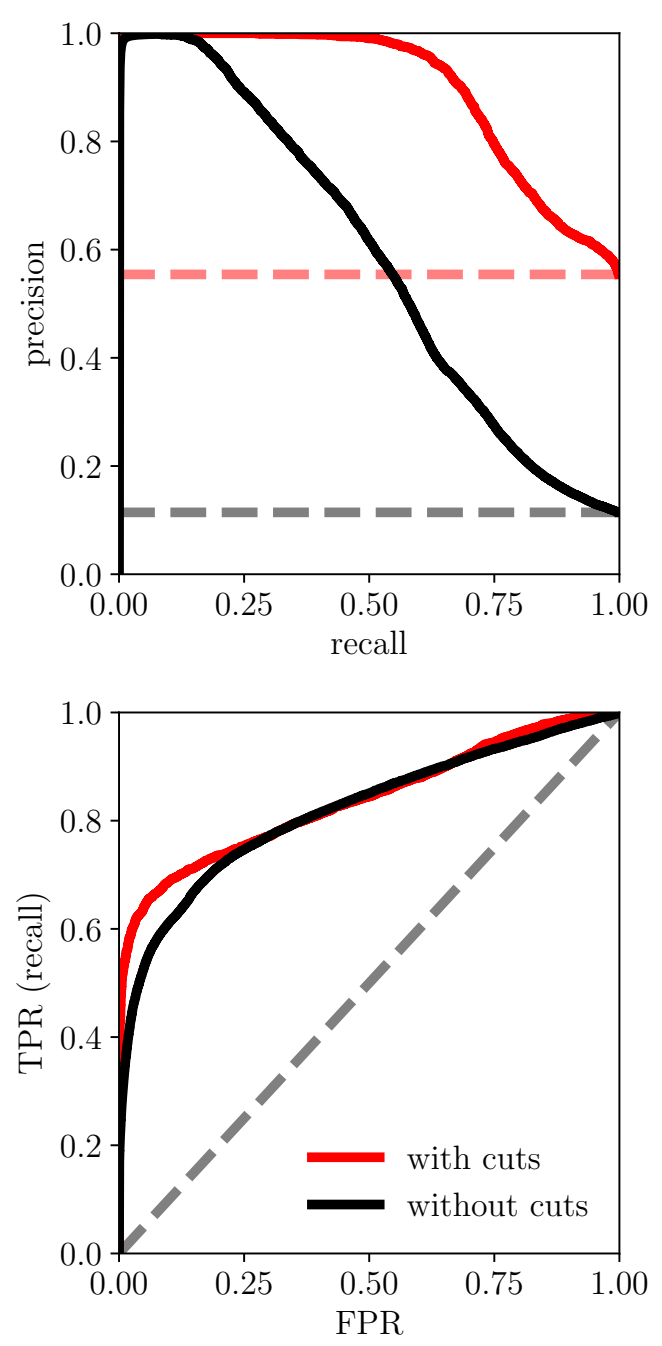

Figure 5. Precision-recall and receiver operating characteristic curves for our algorithm applied to the Kepler data before and after making cuts. In both plots, the dashed lines show the expected performance of a classifier which flags light curves at random.

we manually determined the true period for each signal since an alias had been flagged in many cases. For some signals, the flagged periodic signal was induced by a short-duration nonperiodic one-off event. Although it was not designed to recover such signals, weirddetector is sensitive to them, since a high amplitude one-off event will still produce an excursion away from the baseline flux in the candidate signal for a folded phase curve.

We manually vetted each of these signals with three false positive tests (see Section 4.5), yielding a total of 32 signals that survived as candidates (hereafter weird objects of interest, or WOIs). Of the 33 WOIs, 18 are periodic signals that appear to be caused by neither transiting bodies nor stellar rotation (Section 4.1), 6 are single or double transitlike events (Section 4.2), and 5 are "brightening" events resembling inverted transits (Section 4.3). The remaining 4 WOIs (including Boyajian's star; Boyajian et al. (2016)) are already reported in the literature (Section 4.4). While many of these signals do not satisfy our periodicity assumption
Table 1. Candidate signals that may be caused by heartbeat tides. a flagged period: 45.5543(91)

\begin{tabular}{lll}
\hline \hline KIC & $P$ [days] & $\zeta$ \\
\hline \multicolumn{3}{l}{ High confidence } \\
\hline 4371947 & $19.7175(39)$ & 926.17 \\
5363987 & $47.4229(95)$ & 94.25 \\
6862114 & $27.6799(55)$ & 5197.9 \\
7837214 & $7.4895(15)$ & 1221.98 \\
7870350 & $23.1156(46)$ & 226.8 \\
8694536 & $35.9492(72)$ & 312.45 \\
10737327 & $22.7772(46)^{a}$ & 115.59 \\
11397541 & $13.2541(27)$ & 2572.16 \\
11498661 & $42.0856(84)$ & 27.08 \\
\hline Low confidence & \\
\hline 3750091 & $31.7631(64)$ & 352.57 \\
3849795 & $17.7238(35)$ & 2864.48 \\
4359409 & $23.4415(47)$ & 190.97 \\
4648556 & $25.6132(51)$ & 109.8 \\
5688669 & $5.7852(12)$ & 6100.39 \\
6946895 & $34.2370(68)$ & 28801.39 \\
7364224 & $4.62144(92)$ & 136.02 \\
7581961 & $12.2131(24)$ & 155.09 \\
9514963 & $18.3001(37)$ & 166.52 \\
& & \\
\hline \hline
\end{tabular}

(the single dips and brightening events, we have chosen to report them because similar events are likely to be flagged when applying this method to other data sets.

\subsection{Periodic signals}

We flagged 18 periodic signals with $P<50$ days, which we divided into high-confidence and low-confidence groups of 9 signals each (see Fig. 6, Table ??). The low-confidence group is not distinguished by low $\zeta$ values, but by the presence of stochastic variability with timescales comparable to those of their candidate periodic signals (see Figure 7 for contrasting examples of each). Given the finite Kepler baseline, we do not want to rule out the possibility that signals such as those in our low-confidence group could spuriously arise.

We hypothesize that a plausible interpretation for many of the WOI periodic signals is that they are systems exhibiting heartbeat tides and in non-eclipsing configurations. Heartbeat binaries (which are named for the fact that their light curves often resemble electrocardiograms) are a class of highly eccentric binaries that display growing and shrinking ellipsoidal variations over the course of an orbit (Welsh et al. 2011; Thompson et al. 2012). The light curves of heartbeat binaries display a variety of temporal morphology not present in our flagged sample, but it's possible that this is caused by a detection bias of our algorithm or of the cross-matched catalogs. For example a heartbeat binary that exhibits a periodic decrease in flux, rather than an increase, is much more likely to be a Kepler threshold crossing event (TCE), and thus thrown out by us.

Penoyre \& Stone (2018) recently highlighted the possibility that heartbeat tides could be caused by highly eccentric massive planets, and our smaller signals $\left(\delta F \approx 5 \times 10^{-} 4\right)$ are in the regime that they calculate could be caused this 
high confidence
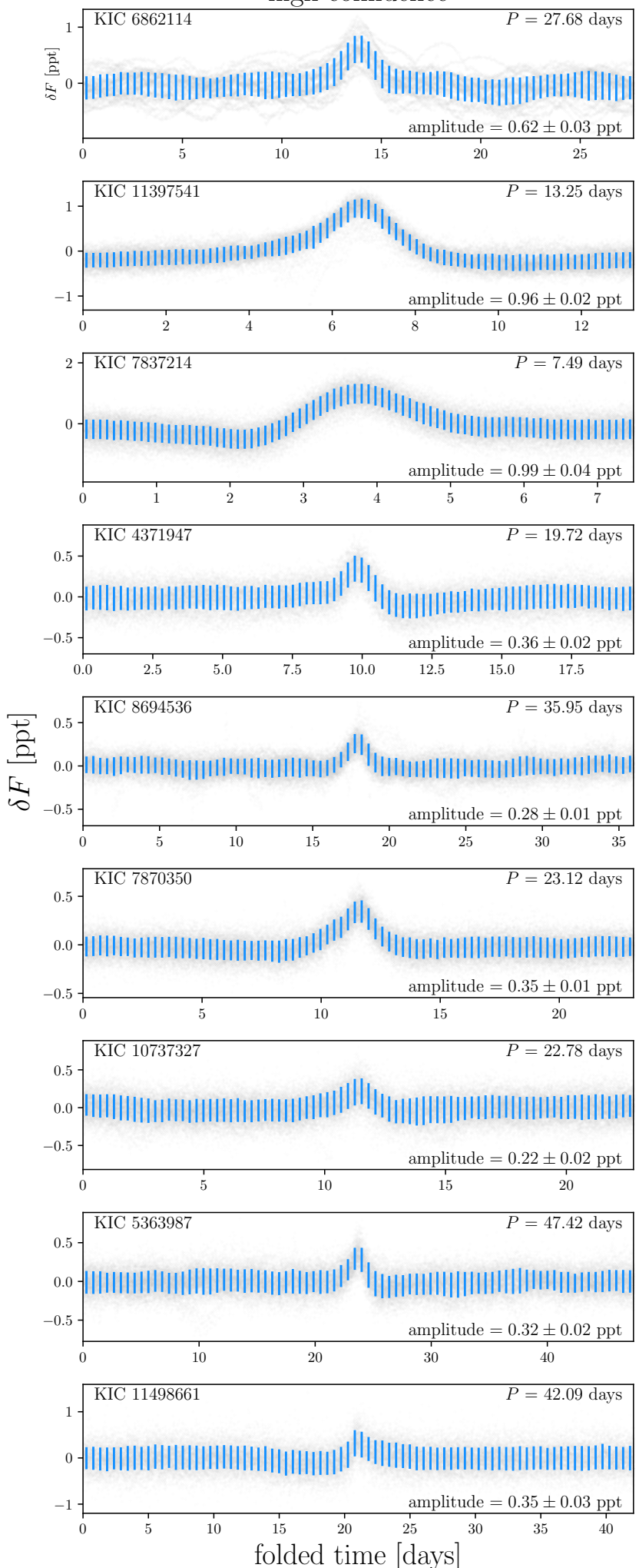

low confidence
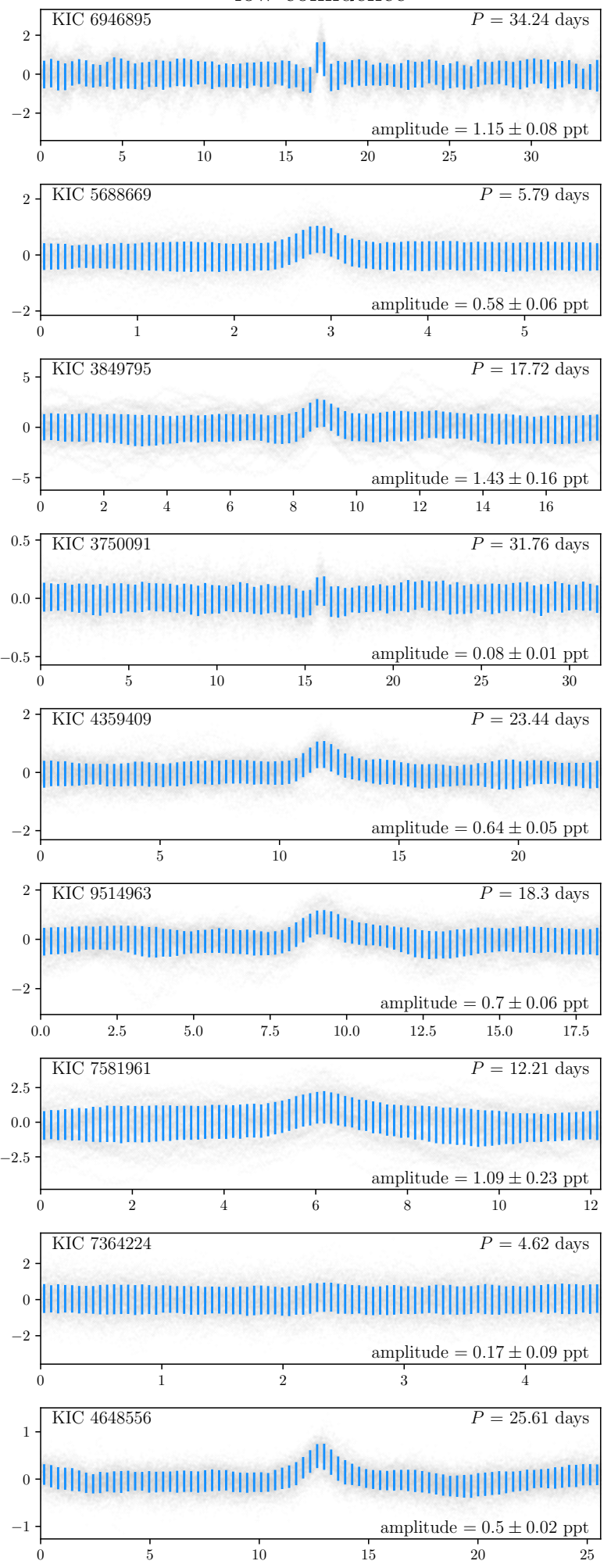

folded time [days]

Figure 6. Eighteen previously unknown periodic Kepler signals. left: the high-confidence detections, right: the low-confidence detections, for which the timescale of stellar variability is roughly that of the flagged signal. The vertical blue lines show the mean and scatter of flux values in 80 constant-width bins in phase. The light curves have not had long-term trends removed. 
KIC 11397541 (high confidence)
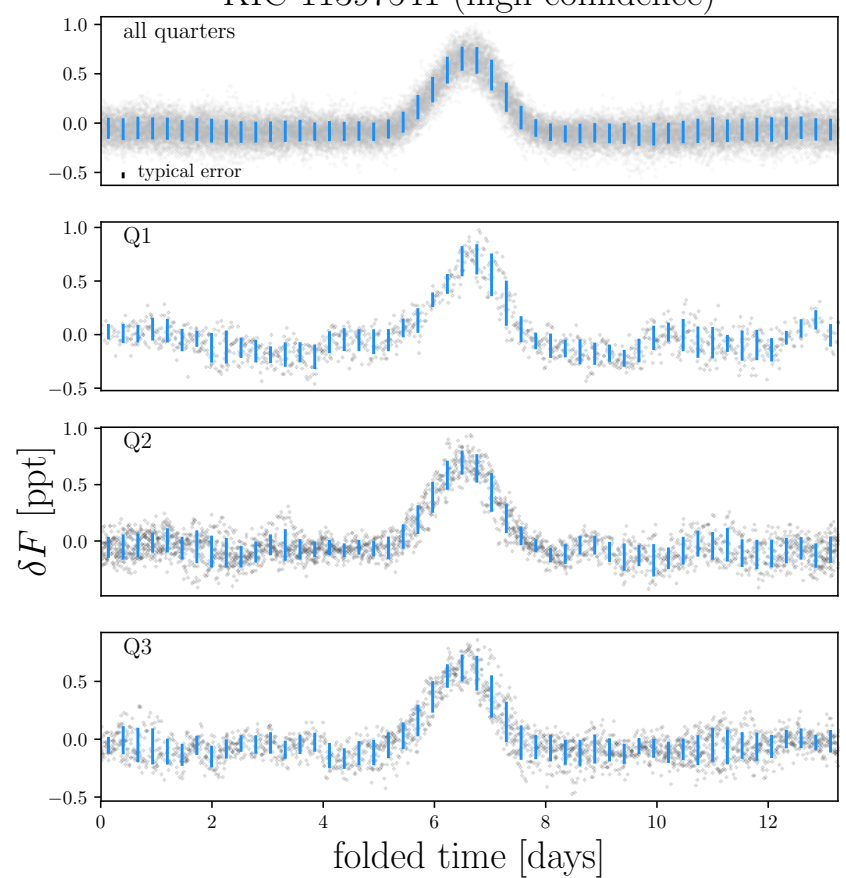

KIC 5688669 (low confidence)
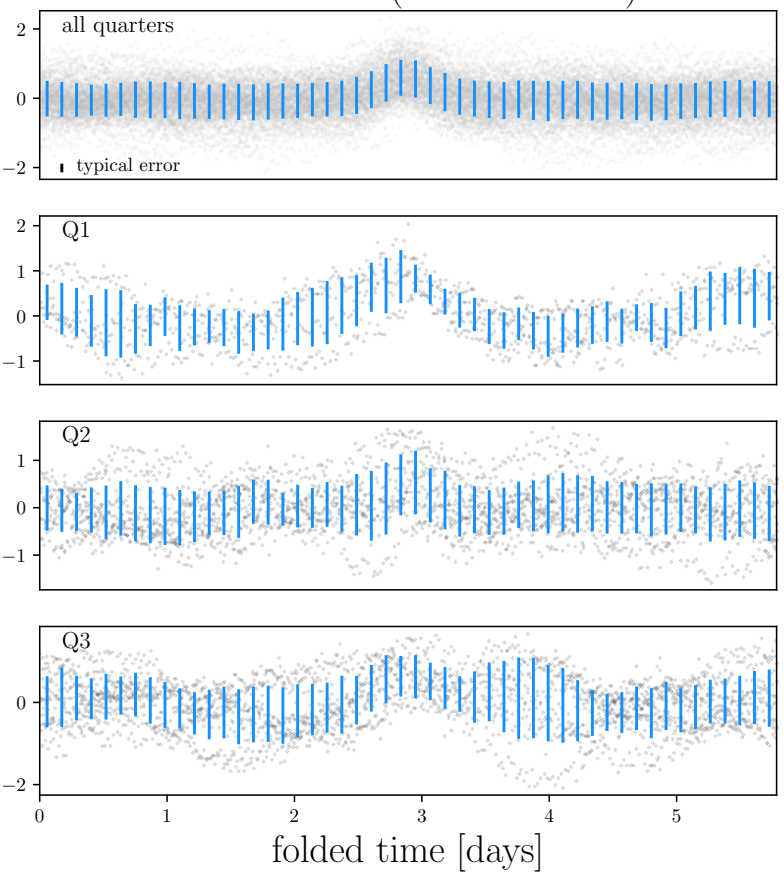

Figure 7. An example of a high-confidence (KIC 11397541) and low-confidence (KIC 11397541) periodic candidate signals, along with phase-folded light curves from quarters 1-4. While both signals appear to be real when folded on all Kepler data, The phase-folded light curves for KIC 11397541 reveal that this signal is not clearly present in the data, but may be an artifact of the finite Kepler baseline.

way. We emphasize, however, that if these signals are indeed caused by heartbeat tides, stellar companions are generally more likely to be the culprit. In order to calculate the amplitude of each signal (reported in Figure 6), we fit it with a sum of harmonic oscillations with periods $P, \frac{P}{2}, \ldots, \frac{P}{30}$, where $P$ is the period of the signal.

These signals are coherent over the 1344 days of $K e$ pler's first sixteen quarters, a feature which strongly suggests they aren't induced by the combination of starspots and stellar rotation (Giles et al. 2017). Furthermore, they generally have a very short duty-cycle, e.g. they are "on" in a small fraction of total phase - a feature that which is unlikely to be produced by rotation with persistent bright or dark surface features.

\subsection{Single and double transit candidates}

To characterize our four single and two double-transit WOIs, we used all data within 0.7 days of the transit midpoint, with no detrending. We generated transit models with batman (Kreidberg 2015), and performed the inference with MultiNest (Feroz \& Hobson 2008; Feroz et al. 2009, 2013). All transits were fit jointly with a linear baseline to account for long-term trends. We sampled from quadratic limb-darkening laws using the uninformative parametrization from Kipping (2013). We calculate the log-likelihood by treating measured fluxes as drawn from independent heteroscedastic normal distributions centered on their true values. Specifically,

$\log (\mathscr{L}(\theta))=-\sum_{i} \frac{1}{2}\left(\frac{F_{i}-\mu_{i}(\theta)}{\sigma_{i}}\right)^{2}+C$ where $f_{i}$ and $\sigma_{i}$ are the flux and photometric error of the $i^{\text {th }}$ point, $\mu_{i}(\theta)$ is the flux value at the phase of the $i^{\text {th }}$ point as calculated by batman for the parameters $\theta$, and $C$ is a constant independent of $\theta$. Our MultiNest output, including posterior samples, as well as detailed prior documentation, is available online at this $\mathrm{URL}^{2}$.

All four single dips favor grazing transit geometries, and display excellent fits as seen in Figure 8. Consequentially, their impact parameters, $b$, are nearly unconstrained from above and are in practice limited by the arbitrary maximum value set by our prior $\left(b_{\max }=1.5 R_{\star}\right)$. This truncation of parameter space effects posterior estimation of $R_{P} / R_{\star}$, which is strongly positively covariant with $b$, and means that we can only provide lower bounds on planetary radii. To derive these bounds, we combine samples from $p\left(R_{P} / R_{\star} \mid\right.$ data $)$ for each orbiting body with the value of $R_{\star}$ reported in Mathur et al. (2017), which is reported as a single value with upper and lower error bars. Given $R_{\star}=\mu_{-\sigma_{-}}^{+\sigma_{+}}$, we adopt a density of

$p\left(R_{*} \mid \mu_{-\sigma_{-}}^{+\sigma_{+}}\right)= \begin{cases}2 \frac{\sigma_{-}}{\sigma_{-}+\sigma_{+}} \mathscr{N}\left(R_{*} \mid \mu, \sigma_{-}\right) & R_{*} \leqslant \mu \\ 2 \frac{\sigma_{+}}{\sigma_{-}+\sigma_{+}} \mathscr{N}\left(R_{*} \mid \mu, \sigma_{+}\right) & R_{*}>\mu\end{cases}$

This is equivalent to equation (3) of Yi et al. (2018), save that we are not formally excluding negative stellar radii.

Inferred properties of singly-transiting bodies are typically very uncertain because of the lack of constraining power on the orbital period. We adopt a uniform prior in $\log P$, and a skewed-normal prior on $\rho$ taken from Mathur et al. (2017) with a density of the same form as that given

2 https://github.com/CoolWorlds/WDdata 
Table 2. Six new single and double transiting system candidates. Note that KIC 7947784 displays two transits but is actually likely two distinct orbiting bodies.

\begin{tabular}{|c|c|c|c|c|}
\hline $\mathrm{KIC}$ & $\zeta$ & epoch [BKJD] & $P$ [days $]$ & $R_{P}\left[R_{\odot}\right]$ \\
\hline \multicolumn{5}{|c|}{ Single transits } \\
\hline $\begin{array}{l}4754691 \\
5475628 \\
10190048 \\
10474113\end{array}$ & $\begin{array}{l}307.38 \\
89.07 \\
634.99 \\
433.29\end{array}$ & $\begin{array}{l}1010.63348_{0.000036}^{+0.00036} \\
950.58131_{-0.000035}^{+0.00032} \\
1260.58772_{-0.000073}^{+0.00037} \\
1442.857365_{-0.00036}^{+0.00039}\end{array}$ & $\begin{array}{l}2875_{-557}^{+757} \\
975_{-221}^{+309} \\
1508_{-215}^{+325} \\
2392_{-828}^{+2763}\end{array}$ & $\begin{array}{l}\geqslant 0.206_{-0.075}^{+0.082} \\
\geqslant 0.536_{-0.114}^{+0.109} \\
\geqslant 0.262_{-0.093}^{+0.092} \\
\geqslant 0.568_{-0.212}^{+0.278}\end{array}$ \\
\hline \multicolumn{5}{|c|}{ Double transits } \\
\hline 7947784 & $\begin{array}{l}1303.57 \\
1303.57 \\
1078.21\end{array}$ & $\begin{array}{l}905.25744_{-0.00045}^{+0.00046} \\
1377.40631_{-0.00044}^{+0.00044} \\
261.34493_{-0.00046}^{+0.00044}\end{array}$ & $\begin{array}{l}1451_{-42}^{+53} \\
1270_{-18}^{+38} \\
680.83003_{-0.00062}^{+0.00062}\end{array}$ & $\begin{array}{l}\geqslant 0.223_{-0.040}^{+0.051} \\
\geqslant 0.399_{-0.088}^{+0.120} \\
\geqslant 0.382_{-0.141}^{+0.139}\end{array}$ \\
\hline
\end{tabular}
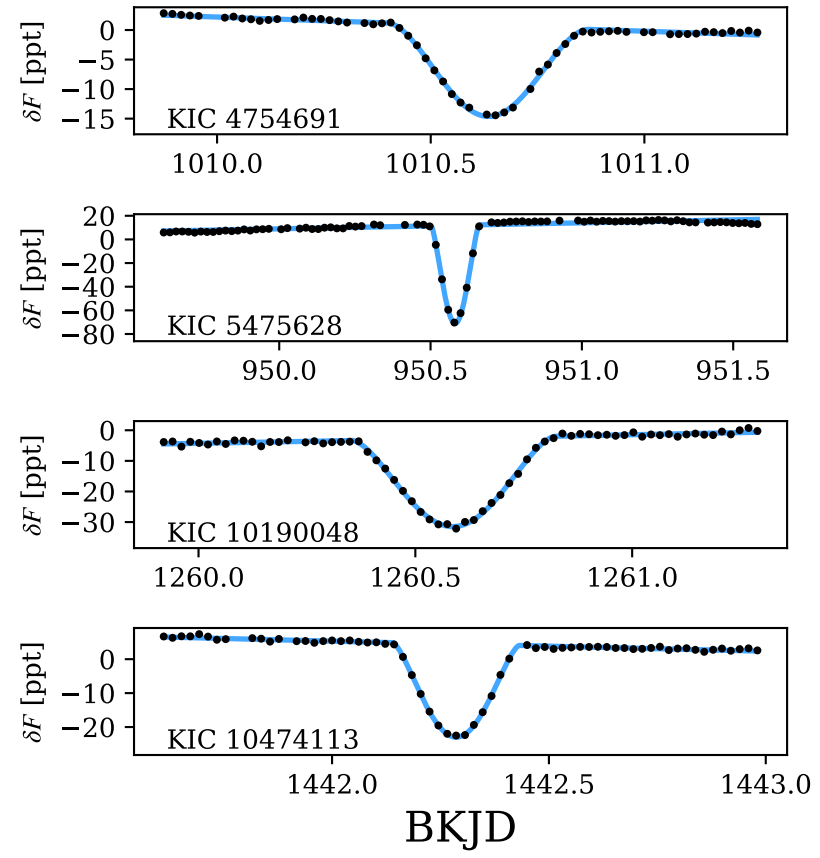

Figure 8. Single transit events, with maximum a posteriori (MAP) fit in blue. Photometric errors are accounted for but too small to be visible.

in Equation (15). The minimum orbital period for the prior was set by using the Kepler light curve itself to check what periods were excluded. Our prior over all parameters not listed here is uniform over a finite window (see the online documentation for details).

We first ran our inference code on all single dips with eccentricity fixed to zero and found that KIC 4754691 and KIC 10190048 were well-described by this model (see Table 2 for the inferred parameters). For KIC 5475628 and KIC 10474113, we find eccentricity is required to explain the shapes, so we allowed eccentricity to vary with uniform priors on $\sqrt{e} \sin (\omega)$ and $\cos (\omega)$ ( $\omega$ being the longitude of periastron).

KIC 8508736 has two transit-like events that are well-fit by a single orbiting body. Because we observe the putative body at two epochs, its orbital period is well-constrained to high precision. The other two transit-like events, in the light curve of KIC 7947784, do not appear to share the same morphology - as evident from the top panel of Figure 9. It is possible that we are seeing two transits of a single body exhibiting extreme precession. However, in this scenario the period would be 472 days and thus should have repeated in quarter 5 (yet clearly does not), unless the precession was so exteme it evades transit for that epoch. We consider it more likely we are witnessing two independent single transits instead and model it as such here.

\subsection{Brightening events}

In total, we detected five KICs displaying brightening events, or inverted transits. However, we find that four of these five WOIs (Figure 11) that passed our false positive tests (KIC 4139302, KIC 4244573, KIC 4244581, and KIC 7340500) are caused by the Solar System comet C/2006 Q1 passing through the Kepler field-of-view. Two signals that were flagged as blends (from KIC 3937417 and KIC 7935479) are also caused by the comet.

The cometary nature of these events is easily recognizable because some of their epochs are separated by a timescale of order days, and they trace out a clear path on the sky. We searched for Solar System bodies by the event ephemeris for one of our signals using JPL's online HORIZONS tool ${ }^{3}$, from which we obtained detailed ephemerides of the comet's path (see Figure 10). Because Kepler is on an Earth-trailing orbit (Van Cleve \& Caldwell 2016), its ephemerides for Solar System objects are different from those of a ground-based observer. This accounts for the systematic offset between out events and the geocentric C/2006 Q1 ephemerides.

The presence of this comet in Kepler was reported in Griest et al. (2014), along with that of C/2007 Q3 and 3 possible new comets, although none of the light curves flagged by our algorithm were among those they reported. A non-exhaustive manual search along the path of the comet yielded three more contaminated light curves not reported in

\footnotetext{
3 https://ssd.jpl.nasa.gov/?ephemerides
} 

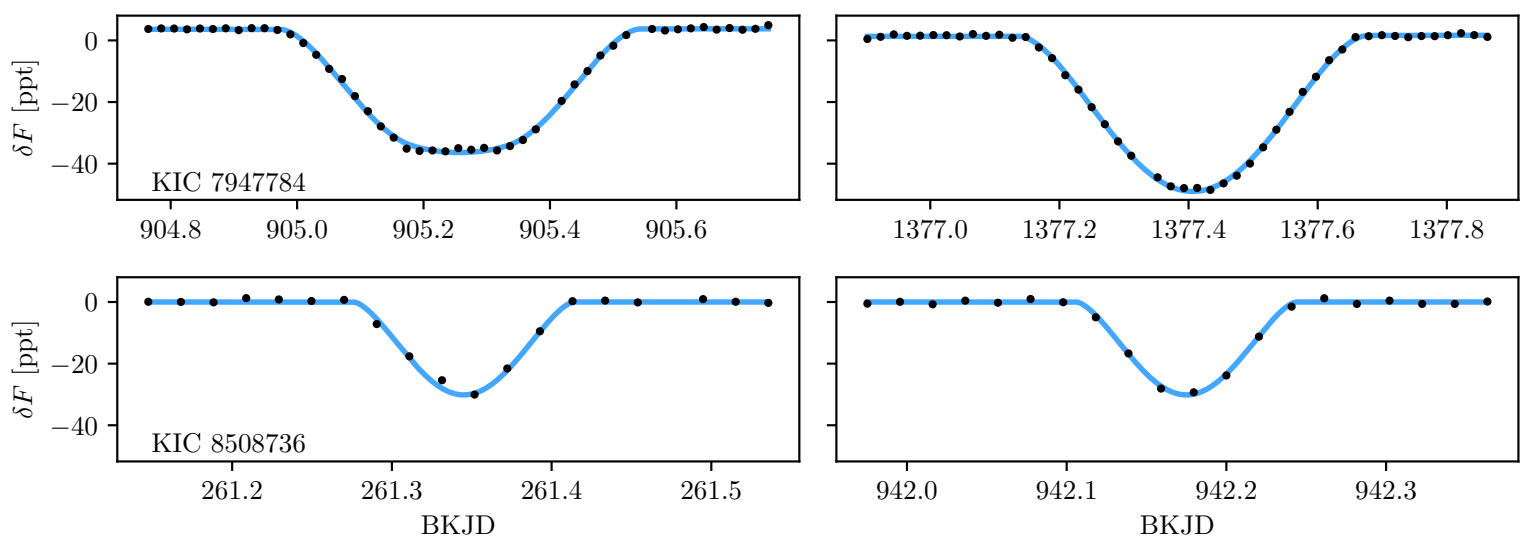

Figure 9. Double transit events, with maximum a posteriori fit in blue. KIC 7947784 was fit with two singly-transiting planets, while KIC 8508736 was fit with a single planet. Photometric errors are accounted for but too small to be visible.

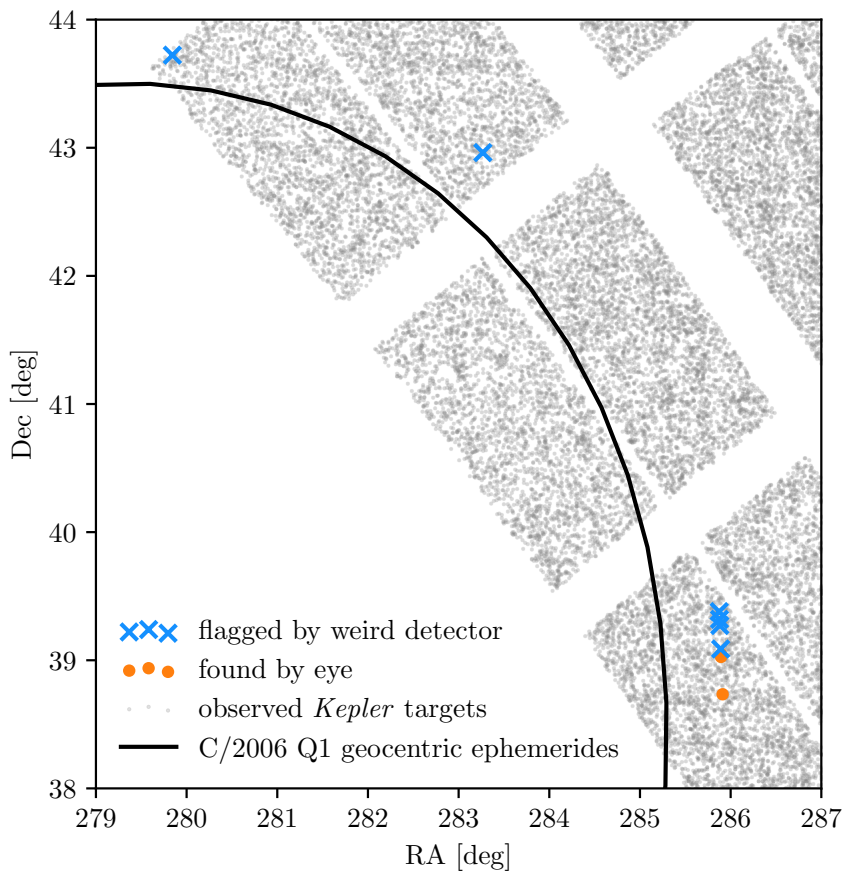

Figure 10. The path of $\mathrm{C} / 2006 \mathrm{Q} 1$ on the sky as viewed by a geocentric observer from HORIZONS, along with the ephemerides of our WOIs and false positives induced by the comet. Kepler's earth-trailing orbit means that it's ephemerides for solar-system objects are offset from geocentric ephemerides.

Griest et al. (2014): KIC 3628785, KIC 3937430, and KIC 3937432. We attribute the fact that we didn't recover the KICs they flagged to our method being designed for the detection of periodic signals, as well as the limitations of manual analysis of flagged events.

The remaining brightening WOI, KIC 5182131, is not associated with C/2007 Q3 and we argue is likely caused by flaring. Flares generally have rise times of a few minutes or less, and have a strongly asymmetrical temporal morphology. Complex flares, which are made up of multiple si-
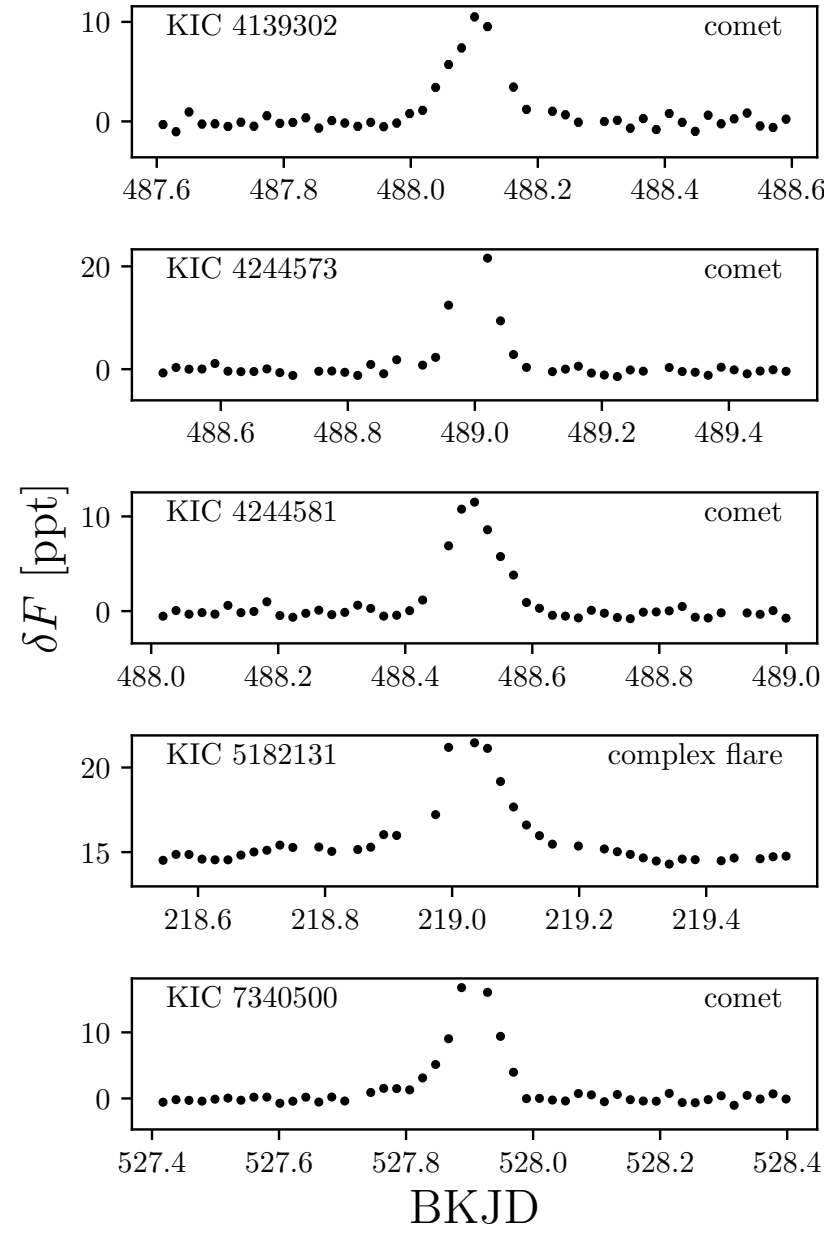

Figure 11. Brightening events. Photometric errors are accounted for but too small to be visible. Four out of five brightening events are caused by passing solar-system comet C/2006 Q1, and one is likely a complex flare. 
multaneous flares, are the exception to this rule and are typically seen only on particularly active stars (Davenport et al. 2014). KIC 5182131 exhibits frequent classical flares, in contrast to the other stars with brightening events, so we hypothesize that its event is a complex flare (see Figure 11). This event is not fit well by a self-lensing binary model, (e.g. Kruse \& Agol 2014), which is equivalent to an inverted transit model when the Einstein radius of the lens is small (Agol 2003).

\subsection{Previously reported signals}

Besides those that are in the catalogs with which we crossmatched, we found four signals that have been previously reported in the literature. We performed the same falsepositive tests on these as for the previously unreported defections. Particularly notable is that fact that we recover KIC 8462852, also known as Boyajian's star, since it was first detected with the Planet Hunters crowd-sourced manual search (Boyajian et al. 2016). KIC 10402660, a potential ultra-short period planet first reported in Jackson et al. (2013), was flagged, as well as KIC 5793963 and KIC 11918466, which failed our false positive tests, but are reported as transit candidates in Tenenbaum et al. (2012).

\subsection{False positives}

To vet the 52 manually picked signals, we performed three tests, yielding 20 false positives (see Table 3). First, we checked if the signal matched the morphology and ephmerides of known KOIs or eclipsing binaries in the Villanova eclipsing binary catalog (similar to the strategy used by Coughlin et al. 2014). Those with a match we presumed to be either a blend of the star with the known KOI or eclipsing binary, or contaminated by video crosstalk (electrical interference).

In the case that no match was found, we manually examined their target pixel data for signs of blending or crosstalk, which are evident when the signal is not coming primary from the center of the aperture, but is offset or present in only a few pixels. If the signal appears inverted in some pixels, we also take this as an indication of crosstalk. Finally, for periodic signals, we also examined the difference in signal amplitude between quarters and take strongly varied amplitudes as indication of contamination.

\section{DISCUSSION}

Our work has introduced a new approach for flagging unusual astrophysical signals in time series data, under the assumption of strict periodicity. We exploit the fact that a strictly periodic signal is coherent with itself when folded upon the correct period, allowing for its recovery via phase dispersion minimization. As a demonstration, we have applied our algorithm (weirddetector) to 161,786 Kepler light curves and uncovered 18 previously unnoticed periodic signals, and several transiting system candidates, as well as some "contaminant" signals from a passing solar-system comet and stellar activity.

The search for unexpected behavior in data is presently not completely automated, since interpretting such signals requires human judgement. Nevertheless, the statistical properties of threshold crossing events (i.e. candidate signals) can be rigorously determined with an automated approach such as ours. For highly irregular signals, which fall within the extremely-broad class of signals to which it is sensitive, our technique is therefore expected to expedite the discovery process by orders of magnitude.

As mentioned though, a current bottleneck in the discovery process of irregular signals is the need for human interpretation of candidates found by weirddetector. We highlight here that it may be possibe to treat weirddetector as a way of feature-engineering a light curve or set of light curves, to produce a currated set of phase curves that serve as the input to a machine leaning algorithm.

The vast majority of the by-eye analysis performed for this paper was to categorize signals as either aligned flares or stellar rotation and to correct the periods of any signals that were flagged as aliases. A machine-learning approach to phase-curve classification could make it possible to construct a nearly-fully-automated pipeline, making this approach more reproducible and even more scalable to large datasets. Such an approach would effectively replace the manual classification of phase curves in this work with an automated system, much like how planetary candidates are often vetted automatically (e.g. Thompson et al. 2018).

\section{ACKNOWLEDGMENTS}

DMK is supported by the Alfred P. Sloan Foundation. Thanks to the Cool Worlds Lab members and for helpful discussions throughout the course of this research. Thanks to our anonymous reviewer for their helpful comments, from the which the paper greatly benefited.

We acknowledge computing resources from Columbia University's Shared Research Computing Facility project, which is supported by NIH Research Facility Improvement Grant 1G20RR030893-01, and associated funds from the New York State Empire State Development, Division of Science Technology and Innovation (NYSTAR) Contract C090171, both awarded April 15, 2010.

\section{REFERENCES}

Abdul-Masih M., et al., 2016, AJ, 151, 101

Agol E., 2003, ApJ, 594, 449

Akeson R. L., et al., 2013, PASP, 125, 989

Arnold L. F. A., 2005, ApJ, 627, 534

Ballard S., 2018, preprint, (arXiv:1801.04949)

Barclay T., Pepper J., Quintana E. V., 2018, preprint, (arXiv:1804.05050)

Barnes J. W., 2009, ApJ, 705, 683

Barnes J. W., Fortney J. J., 2004, ApJ, 616, 1193

Bouma L. G., Winn J. N., Kosiarek J., McCullough P. R., 2017, preprint, (arXiv: 1705.08891)

Boyajian T. S., et al., 2016, MNRAS, 457, 3988

Carter J. A., Agol E., 2013, ApJ, 765, 132

Cortes J., Kipping D., 2018, ApJ, submittied

Coughlin J. L., 2017, Technical report, Description of the TCERT Vetting Reports for Data Release 25

Coughlin J. L., et al., 2014, AJ, 147, 119

Davenport J. R. A., et al., 2014, ApJ, 797, 122 
Table 3. Flagged signals that failed our false positive tests. The reason column states which false-positive test the signal failed (QAM: signal amplitude mismatch between quarters, EM: ephemeris match).

\begin{tabular}{|c|c|c|c|c|c|c|}
\hline $\mathrm{KIC}$ & $\zeta$ & reason & $P_{\text {true }}[$ days] & $P_{\text {flagged }}[$ days $]$ & $\operatorname{epoch}(\mathrm{s})$ [BKJD] & category/explanation \\
\hline 2578184 & 12.17 & blend & & & 171.6 & brightening event \\
\hline 2993038 & 572.64 & blend & & & $551.0,1256.0$ & single/double transit \\
\hline 3937417 & 317.79 & blend & & & 486.5 & comet \\
\hline 4143192 & 290.83 & EM: KIC 3836439 & $1.54035(31)$ & $6.1614(12)$ & & short period planet \\
\hline 4488363 & 1042.88 & blend & & & $1057.0,1433.0,766.0$ & brightening event \\
\hline 5120608 & 677.81 & blend & & & 1441.0 & brightening event \\
\hline 5194255 & 167.66 & QAM & $18.1543(36)$ & $18.1543(36)$ & & nontransit periodic \\
\hline 5462390 & 1015.34 & blend & & & & instrumental \\
\hline 5793963 & 2471.58 & EM: KIC 6953219 & $0.56681(11)$ & $4.53446(91)$ & & short period planet \\
\hline 6044768 & 2029.32 & EM: KIC 6953219 & $0.56680(11)$ & $7.9352(16)$ & & short period planet \\
\hline 6125804 & 3346.49 & EM: KIC 6953219 & $0.56680(11)$ & $8.5020(17)$ & & short period planet \\
\hline 6525462 & 3001.53 & EM: KIC 6367628 & $3.77979(76)$ & $18.8989(38)$ & & eclipsing binary \\
\hline 6606591 & 3409.47 & EM: KIC 6953219 & $0.56680(11)$ & $8.5020(17)$ & & short period planet \\
\hline 6606797 & 943.74 & EM: KIC 6953219 & $0.56681(11)$ & $2.83405(57)$ & & short period planet \\
\hline 7867378 & 30.68 & blend & & & 912.0 & brightening event \\
\hline 7935479 & 35.98 & blend & & & 555.5 & comet \\
\hline 8114216 & 274.7 & QAM & $11.7977(24)$ & $11.7977(24)$ & & nontransit periodic \\
\hline 8694723 & 217.06 & crosstalk & & & 1280.4 & single/double transit \\
\hline 9955874 & 187.74 & pixel sensitivity dropout & & & 895.5 & single dip \\
\hline 11753409 & 486.83 & EM: KIC 11235323 & $19.6702(39)$ & $19.6702(39)$ & & eclipsing binary \\
\hline 11918466 & 1486.27 & blend & $8.0764(16)$ & $48.4584(97)$ & & short period planet \\
\hline
\end{tabular}

Feroz F., Hobson M. P., 2008, MNRAS, 384, 449

Feroz F., Hobson M. P., Bridges M., 2009, MNRAS, 398, 1601

Feroz F., Hobson M. P., Cameron E., Pettitt A. N., 2013, preprint, p. arXiv:1306.2144 (arXiv:1306.2144)

Fischer D. A., et al., 2012, MNRAS, 419, 2900

Foreman-Mackey D., Morton T. D., Hogg D. W., Agol E., Schölkopf B., 2016, AJ, 152

Giles H. A. C., Collier Cameron A., Haywood R. D., 2017, MNRAS, 472, 1618

Griest K., Cieplak A. M., Lehner M. J., 2014, ApJ, 786, 158

Guterman P., Mazeh T., Faigler S., 2015, in Martins F., Boissier S., Buat V., Cambrésy L., Petit P., eds, SF2A-2015: Proceedings of the Annual meeting of the French Society of Astronomy and Astrophysics. pp 277-281 (arXiv:1509.03403)

Huang X., Bakos G. Á., Hartman J. D., 2013, MNRAS, 429, 2001

Huang C. X., et al., 2018, preprint, (arXiv:1807.11129)

Hui L., Seager S., 2002, ApJ, 572, 540

Jacklin S., Lund M. B., Pepper J., Stassun K. G., 2015, AJ, 150, 34

Jackson B., Stark C. C., Adams E. R., Chambers J., Deming D., 2013, ApJ, 779, 165

Jenkins J. M., et al., 2010, in Software and Cyberinfrastructure for Astronomy. p. 77400D, doi:10.1117/12.856764

Jurkevich I., 1971, Ap\&SS, 13, 154

Kipping D. M., 2008, MNRAS, 389, 1383

Kipping D. M., 2010, MNRAS, 408, 1758

Kipping D. M., 2011, MNRAS, 416, 689

Kipping D. M., 2013, MNRAS, 435, 2152

Kipping D. M., Teachey A., 2016, MNRAS, 459, 1233

Kirk B., et al., 2016, AJ, 151, 68

Knutson H. A., Charbonneau D., Noyes R. W., Brown T. M., Gilliland R. L., 2007, ApJ, 655, 564

Korpela E. J., Sallmen S. M., Leystra Greene D., 2015, ApJ, 809, 139

Kovács G., Zucker S., Mazeh T., 2002, A\&A, 391, 369

Kovács G., Bakos G., Noyes R. W., 2005, MNRAS, 356, 557

Kreidberg L., 2015, preprint (arXiv:1507.08285)

Kruse E., Agol E., 2014, Science, 344, 275
Lafler J., Kinman T. D., 1965, ApJS, 11, 216

Llama J., Vidotto A. A., Jardine M., Wood K., Fares R., Gombosi T. I., 2013, MNRAS, 436, 2179

Lomb N. R., 1976, Ap\&SS, 39, 447

Mamajek E. E., Quillen A. C., Pecaut M. J., Moolekamp F., Scott E. L., Kenworthy M. A., Collier Cameron A., Parley N. R., 2012, AJ, 143, 72

Mathur S., et al., 2017, The Astrophysical Journal Supplement Series, 229, 30

O’Donovan F. T., et al., 2006, ApJ, 651, L61

Parks J. R., Plavchan P., White R. J., Gee A. H., 2014, ApJS, 211, 3

Pearson K. A., Palafox L., Griffith C. A., 2018, MNRAS, 474, 478

Penoyre Z., Stone N. C., 2018, preprint, p. arXiv:1803.05917 (arXiv:1803.05917)

Plavchan P., Jura M., Kirkpatrick J. D., Cutri R. M., Gallagher S. C., 2008, ApJS, 175, 191

Pont F., Zucker S., Queloz D., 2006, MNRAS, 373, 231

Rappaport S., et al., 2012, ApJ, 752, 1

Rauer H., et al., 2014, Experimental Astronomy, 38, 249

Sanchis Ojeda R., Winn J. N., Rappaport S. A., 2015, in American Astronomical Society, AAS Meeting \#225, id.408.01.

Scargle J. D., 1982, ApJ, 263, 835

Seager S., Hui L., 2002, ApJ, 574, 1004

Shallue C. J., Vanderburg A., 2018, AJ, 155, 94

Skrutskie M. F., et al., 2006, AJ, 131, 1163

Stellingwerf R. F., 1978, ApJ, 224, 953

Sullivan P. W., et al., 2015, ApJ, 809, 77

Tamuz O., Mazeh T., Zucker S., 2005, MNRAS, 356, 1466

Tenenbaum P., et al., 2012, The Astrophysical Journal Supplement Series, 199, 24

Thompson S. E., et al., 2012, ApJ, 753, 86

Thompson S. E., et al., 2018, The Astrophysical Journal Supplement Series, 235, 38

Van Cleve J. E., Caldwell D. A., 2016, Technical report, Kepler Instrument Handbook. NASA Ames Research Center

Vanderburg A., et al., 2015, Nature, 526, 546

Vidal-Madjar A., Lecavelier des Etangs A., Désert J. M., Ballester 


\section{$14 \quad$ Wheeler 83 Kipping}

G. E., Ferlet R., Hébrard G., 2003, Nature, 422, 143

Wang J., et al., 2015, ApJ, 815

Warner B., Robinson E. L., 1972, MNRAS, 159, 101

Welsh W. F., et al., 2011, The Astrophysical Journal Supplement Series, 197, 4

Whittiker E. T., Robinson G., 1926, The Calculus of Observations. Blackie \& Son, London

Yi J. S., Chen J., Kipping D., 2018, MNRAS, 475, 3090

Zucker S., Giryes R., 2018, AJ, 155, 147

Zuluaga J. I., Kipping D. M., Sucerquia M., Alvarado J. A., 2015, ApJ, 803, L14 